\title{
New ACCESS TECHNIQUE IN VEHICULAR AD-Hoc NETWORKS
}

\author{
Abdel-Mehsen Ahmad, Zouhair Bazzal and Rola Sabra \\ Department of Computer and Communication Engineering, Lebanese International \\ University, Bekaa, Lebanon
}

\begin{abstract}
Vehicle Ad-Hoc Networks was developed to improve the safety and comfort while driving a car. In vehicleto-vehicle (V2V) communication, it is a challenging issue for single-radio devices to switch and operate between the seven DSRC channels allocated by the FCC and to support both safety and non-safety applications.

Thereby, the IEEE 1609WG has contrived the IEEE 1609.4 Multi-channel Operation, a protocol that allows a device to switch channel operation over time so that it can efficiently utilize the multi-channel spectrum. However, this protocol showed some limitations, and this is showed through the low probability of successful transmission for safety messages and through the low throughput achieved for service messages.

This paper proposes a new mechanism, Listening-based Immediate SCH Access (LISA), which enhances the channel utilization, i.e. improving the probability of successful safety packets transmission and increasing the throughput for service packets. Using LISA scheme, the probability of successful transmission has increased by $14 \%$ when the probability of transmission is equal to $80 \%$, and the throughput has increased up to five times compared to the original protocol.
\end{abstract}

\section{KEYWORDS}

Vehicular Communications, IEEE 802.11p, IEEE 1609.4, MAC Protocol.

\section{INTRODUCTION}

Motivated by the increasing number of injuries and deaths caused by car accidents, vehicular adhoc networks (VANETs) was proposed as a solution to reduce the accidents [2] as safety management, provide comfort for its users [1], and avoid traffic congestion [5]. In other terms, the applications of VANET could be divided into two main applications: Safety and non-safety applications. It is characterized by its dynamic topology, mobility modeling, predictable mobility patterns, use of other technologies (like GPS), and finally its unlimited power provided by the car itself [1][3]. However, like any other technologies, VANETs suffer from several technical and socio-economic challenges that could be summarized as follows: lack of an online centralized management entity which may lead to packets collision, use of different communication protocols by the car brands, having an efficient routing protocol, and finally security and privacy concerns [1][4].

After knowing a general idea about VANETs, their applications and their challenges, the rest of this paper is structured as follows: The second section is an overview of the standards and protocols used for VANET. In section three, several approaches are discussed concerning the multi-channel operation. The fourth section is about the proposed scheme. Section five includes the simulations and performance analysis. And finally, this paper is concluded in section six, and future work ideas are introduced.

DOI: $10.5121 /$ ijwmn.2017.9306 


\section{Standards ANd Protocols Overview}

In this paper, the focus is on IEEE 802.11p Wireless Access in Vehicular Environments (WAVE) amendment and its extension IEEE 1609.4 standard. The US Federal Communication Commission (FCC) allocated $75 \mathrm{MHz}$ bandwidth of the licensed spectrum in the $5.9 \mathrm{GHz}$ band for Dedicated Short-Range Communication (DSRC); it starts from $5.850 \mathrm{GHz}$ and ends at $5.925 \mathrm{GHz}$ [4]. This band is divided in seven $10 \mathrm{MHz}$ channels (Figure 1a); six of them are service channels $\mathrm{SCH}$ used for non-safety applications, and the remaining channel is control channel $\mathrm{CCH}$ used for safety applications.

In the time division concept shown in Figure 1b, the time is divided into Synchronization periods 'sync periods' (SI). The first 50ms of SI is control channel interval (CCHI) with $4 \mathrm{~ms}$ guard interval at the beginning, and the last $50 \mathrm{~ms}$ is service channel interval (SCHI) with $4 \mathrm{~ms}$ guard interval at the beginning.
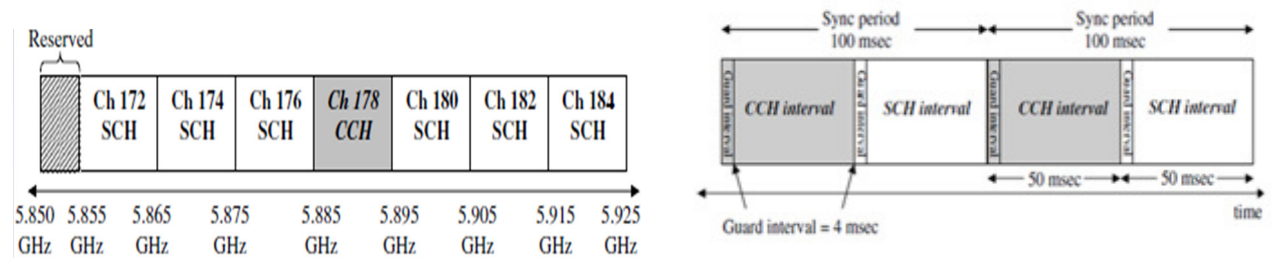

Figure 1. (a) US DSRC band plan channel designations [4]; (b) Division of time into CCH intervals and $\mathrm{SCH}$ intervals [4]

During CCHI, many devices switch to $\mathrm{CCH}$ for rendezvous. During SCHI, a few devices tune to one of the SCHs. What worth mentioning is that only two types of messages are allowed on $\mathrm{CCH}$ while IP packets are not allowed. Those types are:

- WAVE short messages (WS

- M): they are the basic safety messages transmitted from one vehicle node to another.

- WAVE service advertisements (WSA): they are used to declare that one or more WAVE services are available on the SCHs during the next SCHI.

One challenging issue here is how the device should select the SCH that will be noticed in the WSA, with a high likelihood to not be occupied. There are four types of channel accesses: Continuous access, alternating access, immediate SCH access and extended SCH access. In IEEE 1609.4 standard, the normal channel access mode is the alternating service channel where any device will switch at the end of the $\mathrm{CCH}$ interval and returns to the $\mathrm{CCH}$ at the start of the next $\mathrm{CCH}$ interval. However, IEEE 1609.4 provides for an Immediate $\mathrm{SCH}$ access which allows immediate communications access to the $\mathrm{SCH}$ without waiting for the next $\mathrm{SCH}$ interval. It also provides for an Extended SCH access which allows communications access to the SCH until service delivery is completed without pauses for $\mathrm{CCH}$. The other challenging point is how the device can tune to the $\mathrm{SCH}$ during a $\mathrm{CCH}$ interval and meeting at the same time the safety requirements, i.e. to be aware of the safety messages transmitted during the $\mathrm{CCH}$ interval.

As a summary, one of the advantages of the IEEE 1609.4 multi-channel switching protocol is allowing a device with a single radio to support both safety applications and non-safety DSRC services. This might be helpful for marketing to the VANET technology. Also, VANETs using DSRC technology allow collision avoidance between two vehicles equipped with DSRC devices. However, having a single radio is a challenging issue if devices want to switch between channels. 
Also, IEEE 1609.4 multi-channel switching protocol suffers from bandwidth wastage because almost the half of the $\mathrm{CCH}$ bandwidth cannot be used for carrying critical safety messages.

\section{RELATED WORKS}

One of the key challenges that face VANETs is defining a reliable protocol that uses the multichannel spectrum efficiently. And since the main VANET's application is the safety management that aims directly to prevent vehicle accidents, it is essential that this protocol ensure that the safety messages arrive as soon as possible to all the surrounding vehicles without any delays, collisions or transmission errors. And beside all that, this protocol must permit the best switching mechanism allowing the vehicles to be on the $\mathrm{CCH}$ to hear or to broadcast a safety message. As a result, many efforts have been made to achieve those goals, to optimize the performance and QoS of VANETs and to improve the channel utilization.

Starting with VEMMAC scheme [11], it suffers from a high delay when the sender and the receiver negotiate to select the best channel for data transmission. Also, it doesn't consider the life time of the safety message that must be broadcasted if the data transmission is extended to the next CCHI, along with the congestion and collision of messages that occur at the beginning of the $\mathrm{SCHI}$ or CCHI.

An enhanced version of VEMMAC, Dang et al. also proposed an efficient and reliable MAC protocol for VANETs, named VER-MAC [12], which allows broadcasting safety messages twice, $50 \mathrm{~ms}$ apart, to increase the safety broadcast reliability and to avoid collision at the beginning of the CCHI and SCHI. However, the second delayed broadcast has a huge disadvantage on the performance of VER-MAC, and this is showed in the simulation results, where the delay is high for emergency messages. Continuing with the schemes done by Dang et al. [13] [14] [15], they are based on TDMA and CSMA protocols. HER-MAC [13] and CER-MAC [14] allow the vehicles to access the $\mathrm{CCH}$ and broadcast safety messages even when a non-safety message is being transmitted on the SCHs during the $\mathrm{CCH}$ interval (CCHI). However, these approaches have a problem regarding the slots (EmgSlots and SerSlots). Safety and non-safety messages might be lost in case of high vehicle density, where there will be high number of messages that are needed to be transmitted or broadcasted. Eventually, no matter how many EmgSlots and SerSlots

exist; there are not enough for these messages.

Mao and Shen proposed a based slot allocation scheme called the Semi-Greedy (S-G) Scheme (2015) [16]. S-G scheme could be applied along with the TDMA-based methods used is other approaches. It is supposed to be a solution for the wasted slots in TDMA-based schemes. The results showed that S-G scheme improved the use of the $\mathrm{CCH}$, where the slots are fully used. But this scheme is only restricted to the cases where the number of slots in a frame is greater than the number of nodes in a two-hop set; i.e. this scheme could be only applied in rural or in a highway where the vehicle density is low.

The authors of [17] proposed an adaptive multi-channel approach for congestion control in vehicular safety communications. Depending on the current communication traffic condition on $\mathrm{CCH}$ and $\mathrm{SCH}$, and as its name refer, this approach allows a flexible usage of the multi-channel. The results of the simulation showed that the proposed approach, under a high-density environment, outperformed the alternating access in both metrics. But, under a low-density environment, the continuous access outperformed the proposed approach in the average packet delay. 
The authors of Merak [18], an adaptive channel coordination mechanism for vehicular ad-hoc networks, thought about measuring the optimal length of the CCHI and SCHI. But, Merak overlooked the life time of the WSMs that are cached in the queues and that might expire before they get broadcasted. Also, Merak doesn't give a higher priority for the critical emergency messages.

\section{Proposed Scheme}

\subsection{Problem Statement}

As mentioned before, having a single radio is a challenging issue if devices want to switch between channels. Therefore, there should be a mechanism that allows the vehicle nodes to switch to the same channel and to find each other, giving a higher priority to the critical safety messages, to improve the channel utilization, to avoid the collision of safety messages and to increase the throughput for service data. Also, IEEE 1609.4 multi-channel switching protocol suffers from bandwidth wastage; because in some cases where there are no safety messages being broadcasted during CCHI while the vehicle is listening to the $\mathrm{CCH}$. Same thing during the SCHI while the vehicle is listening to the $\mathrm{SCH}$ and there is no data transmitted.

\subsection{System Model}

\section{a) Motivation}

In 1609.4-2010, channel access options include continuous access, alternating service channel and $\mathrm{CCH}$ access, immediate $\mathrm{SCH}$ access, and extended SCH access. But those access operations define only the data format and operating mechanism and they lack specific switching mechanisms so it doesn't limit the time to tune to other channels. Therefore, immediate and extended options may be combined in a single access. Immediate $\mathrm{SCH}$ access allows immediate communications access to the SCH without waiting for the next SCH interval, while Extended $\mathrm{SCH}$ access allows communications access to the $\mathrm{SCH}$ without pauses for $\mathrm{CCH}$ access. So, the proposed schemes are based on these two modes. The proposed mechanism tends to solve the problem of $\mathrm{CCH}$ bandwidth wastage, and the problem of losing safety messages that are being broadcasted on $\mathrm{CCH}$ while the vehicle is on $\mathrm{SCH}$.

\section{b) Proposed Scheme: Listening-Based Immediate SCH Access (LISA)}

The IEEE 1609.4 protocol declares that the total period is $100 \mathrm{~ms}$, where $50 \mathrm{~ms}$ for $\mathrm{CCH}$ and $50 \mathrm{~ms}$ for SCH. As mentioned before, the IEEE Standard 1609.4-2010 permits to vehicles to stay on the $\mathrm{SCH}$ during the $\mathrm{CCH}$ interval. The main challenge here is how this can be done without losing any safety packets that can be transmitted on the $\mathrm{CCH}$ during this period. Also, what should be done in order not to lose non-safety packets transmitted during $\mathrm{CCHI}$ while the vehicle is on $\mathrm{CCH}$ and no safety messages are being broadcasted? In our solution, like CSMA protocol, we define a listening period (LP). This period has the role to determine if there is any safety or data packets will be transmitted during CCHI or SCHI. It always occurs on $\mathrm{CCH}$. 


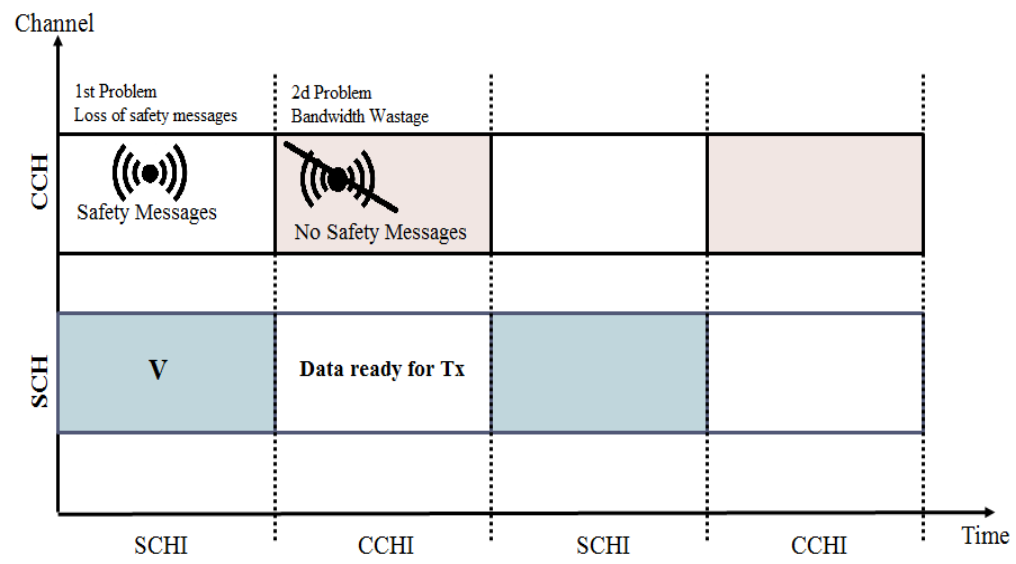

Figure 2. Problems Solved by LISA

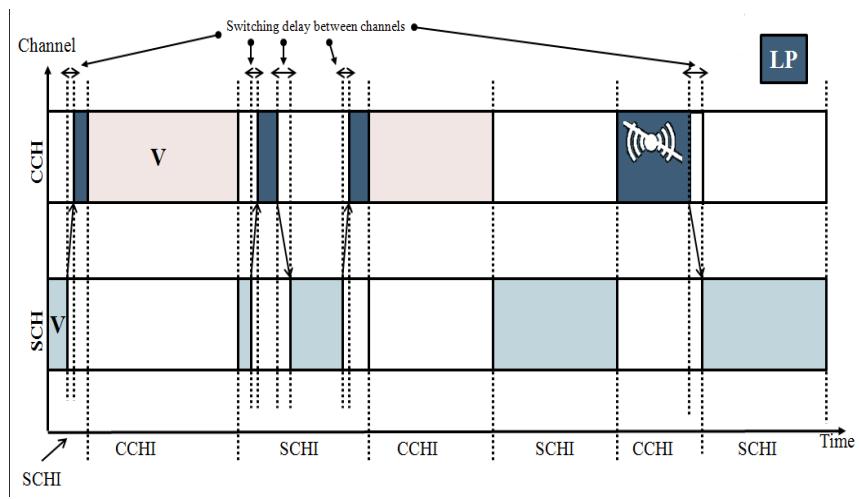

Figure 3. LISA scenario

During the CCHI, if any vehicle wants to stay on the $\mathrm{SCH}$, it listens to the $\mathrm{CCH}$ during the LP. If it senses any signal or noise, it knows that there is a safety messages that can be sent and so it remains on the $\mathrm{CCH}$. If not, the vehicle will go back to $\mathrm{SCH}$ to receive data packets. Also, during SCHI, "V" might go back to $\mathrm{CCH}$ and listen several times to make sure that there is no safety message broadcasted. On the other hand, while $\mathrm{V}$ on $\mathrm{CCH}$ during $\mathrm{CCHI}$ listening if while there are safety messages broadcasted, "V" will tune to the SCH if the LP isn't noisy. And if not, "V" will stay on $\mathrm{CCH}$. By that, LISA compromises between safety messages and data packets. In the next section, the LP is fixed at the beginning and the middle of the SCHI; Its width is equal to the duration needed for one safety packet to be sent and received by the listening nodes.

\section{Simulation Results And Performance Analysis}

\subsection{Simulation Model}

With the purpose of observing the performance of the proposed methods, discrete event-driven simulations are made in MATLAB. In other terms, the DSRC protocol process and the proposed process are coded depending on their behavior using discrete events in time. The parameters used in these simulations are shown in Table 1. 
International Journal of Wireless \& Mobile Networks (IJWMN) Vol.9, No.3, June 2017

Table 1. Simulation parameters

\begin{tabular}{ll}
\hline Parameter & Value \\
\hline Data rate & $6 \mathrm{Mbps}$ \\
Communication range & $1 \mathrm{Km}$ \\
Number of vehicles & $50-350$ \\
Safety packet size & vehicles \\
Non-safety packet size & 100 Bytes \\
Sync interval & 800 Bytes \\
CCH interval & $100 \mathrm{~ms}$ \\
SCH interval & $50 \mathrm{~ms}$ \\
Slot time & $50 \mathrm{~ms}$ \\
Number of runs & $13 \mu \mathrm{s}$ \\
& $50 \mathrm{runs}$ \\
Road model & Highway \\
& $1 \mathrm{Km}$ long, 2 \\
\hline
\end{tabular}

Nevertheless, the performance metrics evaluated are:

\section{- Efficiency For Safety Packets}

In other terms, it is the percentage of successfully transmitted packets to the vehicles listening to $\mathrm{CCH}$. The following equation represents how the efficiency is calculated:

$$
\text { efficiency }=\frac{\text { Number of received messages }}{\text { Total number of transmitted messages }} \times 100
$$

\section{- Throughput For Service Packets.}

These metrics are observed in different scenarios; in other terms, when the number of vehicles increases and when the probability of transmission increases. Then, in the later sub-sections, a comparison is made between these approaches and the original approach i.e. IEEE 802.11p/WAVE protocol. What worth mentioning, is that the slots are allocated randomly, and it is assumed that each car sends service packets and broadcasts safety packets for one time only. Also, the size of the service packets and safety packets are fixed at 800Bytes and 100Bytes respectively.

\subsection{Results And Performance Analysis}

As mentioned before, the LP is fixed at the beginning and at the middle of the SCHI. Its width is equal to the duration needed for one safety packet to be sent and received by the listening nodes. Also, the simulation time is equal to $500 \mathrm{~ms}$ i.e. the simulation is made for 50 sync intervals. While testing the performance of this scheme, four cases were taken into consideration:

CASE 1: It's when the vehicle listens to the $\mathrm{CCH}$ at the beginning and at the middle of the SCHI, and switches back to $\mathrm{SCH}$ since there are no safety packets that are being broadcasted.

CASE 2: It's when the vehicle listens to the $\mathrm{CCH}$ at the beginning, and stays in the $\mathrm{CCH}$ since there are safety packets that are being broadcasted.

CASE 3: It's when the vehicle listens to the $\mathrm{CCH}$ at the beginning of the SCHI, then tunes back to $\mathrm{SCH}$ to receive or send service packets, then tunes back to $\mathrm{CCH}$ in the middle of the SCHI, and stays in $\mathrm{CCH}$ to receive the broadcasted safety packets. 
CASE 4: It's when the vehicle is in the $\mathrm{CCH}$ during $\mathrm{CCHI}$, and there are no broadcasted safety packets for a period equal to two times the sending time required for a safety packet to reach its destination.

In the following we show the performance of LISA approach compared to the IEEE 802.11 $/$ /WAVE, taking into consideration three values for the probability of transmission $\mathrm{P}: \mathrm{P}=$ $0.6, \mathrm{P}=0.7$ and $\mathrm{P}=0.8$.

\section{a) Efficiency}

Figures $4 \mathrm{a}, 4 \mathrm{~b}$, and $4 \mathrm{c}$ show that when the probability of transmission increases, the performance of the IEEE $802.11 \mathrm{p} /$ WAVE decreases. In other terms, when the probability of transmission increases from 0.6 to 0.7 to 0.8 , the efficiency decreases about $5 \%$ to $11 \%$; while LISA approach, in all the cases, has showed an increase in the efficiency even when the probability of transmission increases.

Figure 4a shows that the efficiency increased about 3\%-6\% when LISA approach is used. Even when the number of nodes increased, the efficiency of LISA approach is still better than the original standard.

When the probability of transmission is equal to 0.7 (Figure $4 \mathrm{~b}$ ), the performance of LISA approach and the performance of the original protocol are similar. However, the performance of LISA still better that the original approach since the efficiency increased by $7 \%$.

The LISA approach shows its best performance when the probability of transmission is equal to 0.8 (Figure 4c), while the IEEE 802.11p/WAVE protocol showed the opposite. The efficiency of LISA approach has increased an average of $11.6 \%$.

\section{b) Throughput}

Despite that LISA approach's main goal is to enhance the probability of successful transmission, but it enhanced in a way the throughput for service packets, which is showed in Figures 5a, 5b and 5c, and especially Figure 6 for the case 4. In the original mechanism (Figure 5a), the throughput started to increase with the number of nodes, and then it began to decrease when the number of nodes reached 100 nodes. This is normal, because when the number of transmitting nodes increases, the number of collision will increase too. In Figure $5 b$ and $5 c$, the throughput of the original mechanism increases drastically for the same reason since the probability of transmission increased to 0.7 and 0.8 . However, the throughput has increased for LISA approach in the first three cases because less vehicles stopped transmitting and switched to the $\mathrm{CCH}$ to listen to the broadcasted safety messages.

Figure 6 shows the throughputs obtained of the case 4 and of IEEE 802.11p/WAVE standard for $\mathrm{P}=0.6, \mathrm{P}=0.7$ and $\mathrm{P}=0.8$, i.e. when there are no safety packets being broadcasted during CCHI. In the original mechanism, the throughput has increased significantly for the reasons mentioned before. But, regarding LISA approach, the throughput has increased drastically with the vehicle density. This is due to the number of vehicles that has switched from the $\mathrm{CCH}$ to the SCH to receive the transmitted service packets. 
International Journal of Wireless \& Mobile Networks (IJWMN) Vol.9, No.3, June 2017
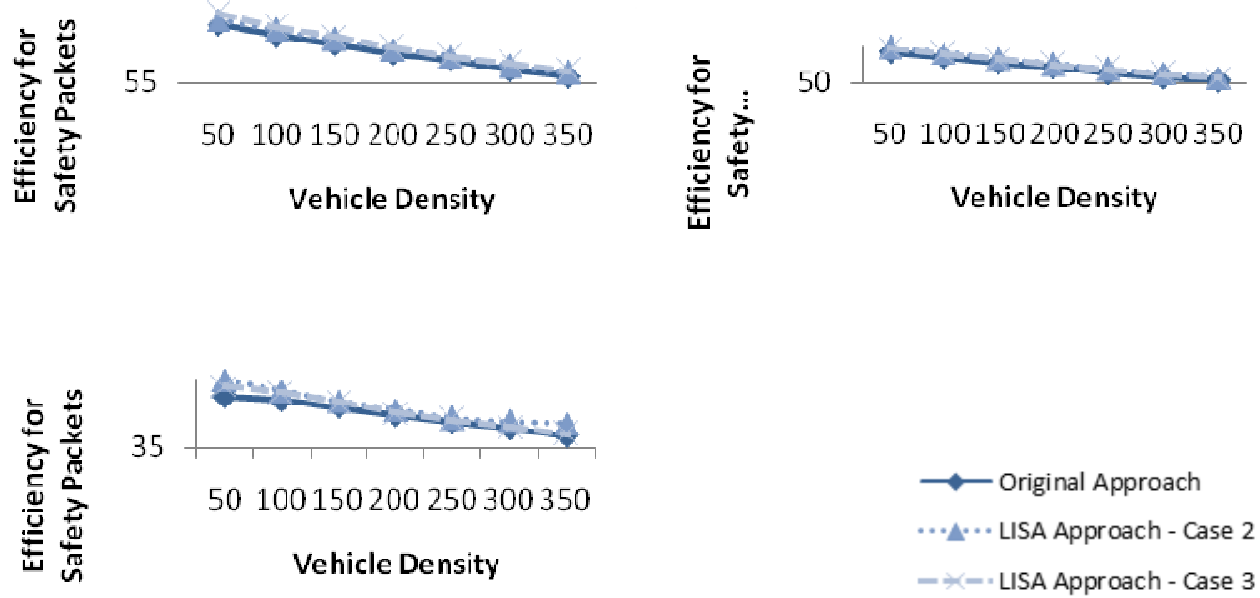

Figure 4. (a) Efficiency vs. Vehicle Density for $\mathrm{P}=0.6$; (b) Efficiency vs. Vehicle Density for $\mathrm{P}=0.7$; (c) Efficiency vs. Vehicle Density for $\mathrm{P}=0.8$

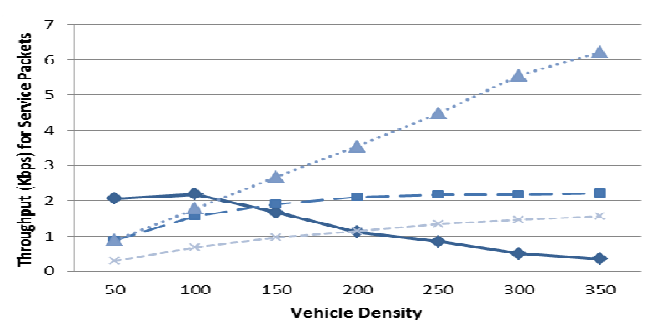

Figure 5. (a) Throughput (Kbps) vs. Vehicle Density for $\mathrm{P}=0.6$;

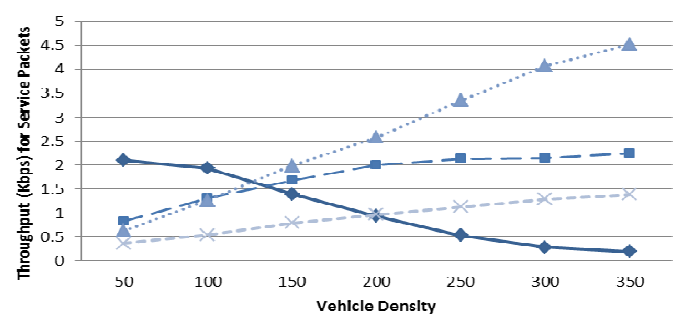

(b) Throughput (Kbps) vs. Vehicle Density for P = 0.7;
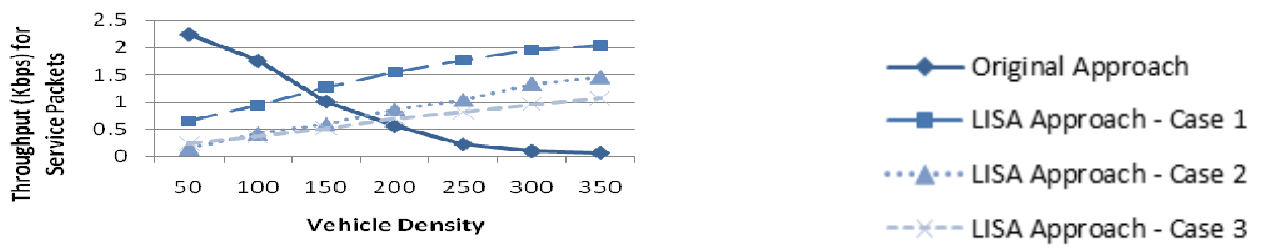

(c) Throughput (Kbps) vs. Vehicle Density for $\mathrm{P}=0.8$
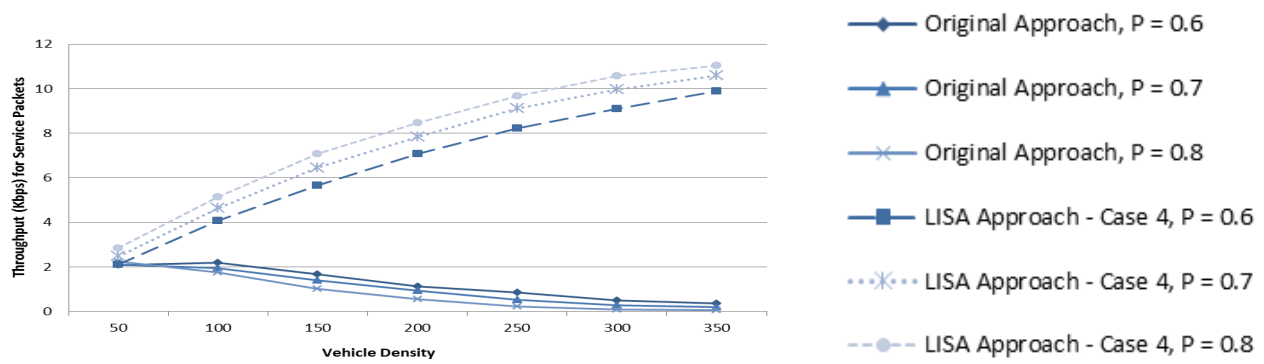

Figure 6. Throughput (Kbps) vs. Vehicle Density for Case 4 
International Journal of Wireless \& Mobile Networks (IJWMN) Vol.9, No.3, June 2017

\section{Conclusion}

This paper proposed LISA approach to enhance the performance of IEEE 802.11p/WAVE protocol. The goal of LISA is increasing the reliability for safety messages transmission in parallel with the throughput for service messages. To achieve that, a vehicle can tune to the $\mathrm{CCH}$ during SCHI for a period, if there are safety packets that are being broadcasted, the vehicle stays in $\mathrm{CCH}$. If not, the vehicle switches back to $\mathrm{SCH}$. Also, during $\mathrm{CCHI}$, if there are no safety packets that are being broadcasted, the vehicle switch to $\mathrm{SCH}$ to receive or send data packets. The MATLAB simulation results indicated that the proposed method can offer efficient channel usage with higher probability of successful safety packets transmission along with higher throughput for service packets. LISA approach has enhanced the probability of successful safety packets transmission by $6 \%, 6.2 \%$ and $14.3 \%$ for probability of transmitting nodes changes from $0.6,0.7$ and 0.8 respectively. Regarding the throughput of service packets, it increased significantly compared with the IEEE $802.11 \mathrm{p} /$ WAVE protocol, where the throughput decreases with the increased probability of transmission and the vehicle density.

\section{REFERENCES}

[1] Rehman, S., Khan, M.A., Zheng, L. and Zia, T. (2013) 'Vehicular Ad-Hoc Networks (VANETs) -An Overview and Challenges', Wireless Networking and Communications, 3(3), pp. 29-38.

[2] Emmelmann, M., Bochow, B. and Kellum, C.C. (2010) 'Vehicular Networking: Automotive Applications and Beyond', 1st edn. UK: A John Wiley and Sons, Ltd, Publication.

[3] Moustafa, H. and Zhang, Y. (2009) 'Vehicular Networks: Techniques, Standards, and Applications', 1st edn., USA: AUERBACH Publications, Taylor \& Francis Group, LLC.

[4] Hartenstein, H. and Laberteaux, P.L. (2010) 'VANET: Vehicular Applications and Inter-Networking Technologies', 1st edn., UK: John Wiley \& Sons Ltd.

[5] Cunha, F., Boukerche, A., Villas, L., Viana, A. and Loureiro, A.F.A. (2014) 'Data Communication in VANETs: A Survey, Challenges and Applications'.

[6] Kenney, J.B. (July 2011) 'Dedicated Short-Range Communications (DSRC) Standards in the United States', Proceedings of the IEEE, 99(7).

[7] Rawashdeh, Z.Y, and Mahmud, S.M. (2011) 'Communications in Vehicular Ad Hoc Networks, Mobile Ad-Hoc Networks: Applications’, Prof. Xin Wang (Ed.), ISBN: 978-953-307-416-0, InTech.

[8] Baumann, R. (2004) 'Vehicular Ad hoc Networks (VANET): Engineering and simulation of mobile ad hoc routing protocols for VANET on highways and in cities', Zurich: ETH.

[9] Jindal, V. and Bedi, P. (March 2016) 'Vehicular Ad-Hoc Networks: Introduction, Standards, Routing Protocols and Challenges', International Journal of Computer Science (IJCSI), 13(2).

[10] Bhoi, S.K. and Khilar, P.M. (2013) 'Vehicular communication: a survey', The Institution of Engineering and Technology (IET Netw), 3(3), pp. 204-217.

[11] Dang, D.N.M., Dang, H.N., Do, C.T. and Hong, C.S. (2013) 'An Enhanced Multi-Channel MAC for Vehicular Ad-Hoc Networks', IEEE Wireless Communications and Networking Conference (WCNC), (1525-3511), pp. 351 - 355.

[12] Dang, D.N.M., Hong, C.S., Lee, S. and Huh, E. (2014) 'An Efficient and Reliable MAC in VANETs', IEEE Communications Letters, 18(4), pp. 616 - 619.

[13] Dang, D.N.M., Dang, H.N., Nguyen, V., Htike, Z. and Hong, C.S., 'HER-MAC: A hybrid efficient and reliable MAC for vehicular ad hoc networks,' IEEE 28th International Conference on Advanced Information Networking and Applications (AINA), pp. 186 - 193, 2014. 
[14] Dang, D.N.M., Dang, H.N., Vo, P.L. and Ngo, Q.T., 'A Cooperative - Efficient - Reliable MAC protocol for Vehicular Ad hoc Networks,' IEEE International Conference on Advanced Technologies for Communications (ATC), 2015.

[15] Dang, D.N.M., Nguyen, V., Chuan, P., Oo, T.Z, and Hong, C.S. 'An efficient and reliable MAC for vehicular ad hoc networks,' 16th Asia-Pacific Network Operations and Management Symposium (APNOMS), pp. 1-6, 2014.

[16] Mao, Y. and Shen, L. (2015) 'Semi-Greedy Scheme for Slot Allocation in Vehicular Networks',Wireless Communications \& Signal Processing (WCSP), 2015 International Conference: IEEE.

[17] Chaitien, K., Tanwongvarl, C. and Chantaraskul, S. (2015) 'Adaptive Multichannel Approach for Congestion Control in Vehicular Safety Communications', IT Convergence and Security (ICITCS), 2015 5th International Conference: IEEE.

[18] Li, M., Liu, C., Wei, D., Zhu, J. and Hou, Y. (2014) 'An Enhanced Distributed Channel Assignment Scheme for VANET', Instrumentation and Measurement, Computer, Communication and Control (IMCCC), Fourth International Conference, pp. 770 - 773: IEEE.

[19] 'IEEE Standard for Wireless Access in Vehicular Environments - Multi-Channel Operation', IEEE Std. 1609.4-2016, Jan. 29, 2016. 